Meta

Journal des traducteurs

Translators' Journal

\title{
Les micro-banques de terminologie
}

Instruments d'une pratique; outils d'apprentissage - Le système

« TERM.X »

\section{Daniel Gouadec et A. Le Meur}

Volume 29, numéro 4, décembre 1984

URI : https://id.erudit.org/iderudit/002943ar

DOI : https://doi.org/10.7202/002943ar

Aller au sommaire du numéro

Éditeur(s)

Les Presses de l'Université de Montréal

ISSN

0026-0452 (imprimé)

1492-1421 (numérique)

Découvrir la revue

Citer cet article

Gouadec, D. \& Le Meur, A. (1984). Les micro-banques de terminologie :

instruments d'une pratique; outils d'apprentissage - Le système " TERM.X ".

Meta, 29(4), 367-374. https://doi.org/10.7202/002943ar d'utilisation que vous pouvez consulter en ligne.

https://apropos.erudit.org/fr/usagers/politique-dutilisation/ 


\section{LES MICRO-BANQUES DE TERMINOLOGIE INSTRUMENTS D'UNE PRATIQUE ; OUTILS D'APPRENTISSAGES LE SYSTÈME «TERM.X »}

D. Gouadec ET A. Le Meur

La domestication de l'informatique doit sans nul doute inciter le traducteur, le rédacteur, le terminographe, mais aussi les enseignants responsables des formations de ces spécialistes, à exploiter l'infinie souplesse d'utilisation des micro-ordinateurs. Face aux géants que sont les banques de terminologie en vraie grandeur, les micro-banques de terminologie semblent devoir se développer en raison des avantages qu'elles ne sauraient manquer d'offrir en vertu de leur extrême souplesse. Les micro-banques de terminologie peuvent ainsi devenir un auxiliaire des pratiques professionnelles et un moyen privilégié des apprentissages.

Les objectifs fixés dans la mise au point de micro-banques de terminologie sont les suivants :

a) possibilité de constitution, modification et gestion de fichiers terminologiques ;

b) possibilité d'exploitation directe et selon des modalités simples des fichiers terminologiques dans la pratique de la traduction, de la rédaction, de la documentation ;

c) possibilité d'exploitation des programmes dans le cadre de la formation des terminographes, traducteurs ou traducteurs-terminographes :

- par initiation à la constitution de glossaires terminologiques,

- par initiation à l'utilisation des données terminologiques.

Les micro-ordinateurs courants permettent, par la mise au point de logiciels spécifiques appelant une étroite collaboration entre terminologues et informaticiens, d'atteindre ces divers objectifs. Le "système terminologique " que nous décrivons ici peut répondre aux besoins les plus divers.

MATÉRIEL

Le matériel utilisé est un micro-ordinateur de type courant avec lecteur de disquettes. Les disquettes utilisées appartenaient, pour les premières, au type "double face " et, pour les secondes, au type "simple face ". La dualité des types de disquettes permettait en effet de tester les capacités de stockage et de gestion dans les deux hypothèses de travail.

\section{LOGICIELS}

Les logiciels mis au point sont au nombre de quatre : 
1. un programme de création de cadre : CRECA.

Ce programme permet à chaque utilisateur de créer un modèle de fiche ou, le cas échéant, plusieurs modèles de fiches. Chacun peut ainsi, puisque le cadre n'est pas préformé, respecter une norme ou un standard ou, au contraire, en fonction de besoins propres, déterminer librement :

- le nombre et la nature des diverses rubriques de chaque fiche,

- la taille de chaque champ,

- le nombre de pages par fiche.

On peut ainsi créer un cadre de fiche qui ne comporterait, par exemple, que les rubriques : VEDETTE, USAGE, SYNONYMES, ÉQUIVALENTS. On peut, sans modification des programmes, créer un cadre de fiches qui comporterait, par exemple, les rubriques : VEDETTE, USAGE, CATÉGORIE GRAMMATICALE, SYNONYMES, ÉQUIVALENTS, DÉFINITION, CONTEXTE, SOURCE, BIBLIOGRAPHIE ANNEXE, DESCRIPTEURS, CORRÉLATIONS, ANNÉE DE CRÉATION DE LA FICHE, AUTEUR DE LA FICHE, etc. On peut créer des fiches sur un écran ou deux écrans ou sur une fraction d'écran. On peut moduler à loisir la place dévolue à chaque type de données.

\section{2. un programme de création de fiches : REMCA}

Une fois le cadre construit, le programme REMCA permet la création du fichier. Le programme lancé, il propose tour à tour les différents champs de la fiche en vérifiant au préalable qu'il n'existe pas déjà une fiche de même vedette. Le programme REMCA permet de :

- constituer les fiches,

- supprimer les fiches existantes,

- modifier des fiches existantes (correction de mots/lettres/etc./insertion ou suppression de mots/lettres/phrases/paragraphes).

Le programme REMCA autorise, sans connexion externe, une remise à jour constante par l'utilisateur lui-même. La seule décision que doive prendre l'utilisateur concerne le nombre et la nature des champs qui seront indexés aux fins de gestion ultérieure.

\section{3. un programme de sortie/listage des fiches : LISCA}

Ce programme permet l'accès à chaque vedette, soit directement si cette vedette est connue, soit indirectement par consultation de la totalité du contenu du fichier. L'interrogation par LISCA peut intervenir par troncature : dans l'hypothèse où l'unité terminologique est polynucléaire et où un seul des éléments constituant cette unité est connu - au moins partiellement - on obtiendra toutes les fiches comportant le " mot " de référence.

\section{4. un programme de gestion du fichier : BASE}

Ce programme se subdivise en quatre sous-programmes :

TB0 : sous-programme de remise à zéro avant constitution d'une base de données,

TB1 : sous-programme organisant le fichier en base de données,

TB2 : sous-programme de gestion du fichier en base de données, et consultation,

TB3 : sous-programme de listage des contenus de la base.

Une fois le fichier constitué en base de données, ce fichier est géré par le logiciel BASE qui procède aux indexations et devient le logiciel d'exploitation du fichier. La constitution en thésaurus est facultative mais possible.

Il est important de signaler que TOUTES LES MANIPULATIONS sont effectuées par l'utilisateur lui-même (traducteur/terminographe/rédacteur/enseignant ou élève). La constitution et la gestion du fichier ne font appel à aucun élément extérieur. Il 
faut également souligner que les corrections sont possibles une fois que le fichier est géré en base de données : elles supposent une manipulation par suppression de fiches et substitution de nouvelles fiches.

\section{UTILISATION DES PROGRAMMES}

L'utilisation des programmes nécessite un apprentissage qui ne devrait guère excéder 12 heures. Une fois cet apprentissage effectué, chaque utilisateur se trouvera en mesure de gérer, en parfaite autonomie, sa ou ses terminologie(s).

L'hypothèse la plus courante d'utilisation des programmes est la suivante :

a) l'utilisateur choisit un ou plusieurs cadres et crée ce(s) cadre(s) à l'aide du programme CRECA ;

b) l'utilisateur constitue son fichier terminologique à l'aide du programe REMCA ;

c) lutilisateur choisit un mode de gestion du fichier :

- gestion minimale par les vedettes à l'aide du seul programme LISCA,

- gestion maximale par tout champ de fiches à l'aide du programme BASE.

Dans le premier cas, le fichier est automatiquement géré par LISCA et se trouve donc exploitable immédiatement. Dans le second cas, le fichier est "entré dans la base".

EXEMPLE DE CONSTRUCTION DE FICHIER TERMINOLOGIQUE : TERMX

L'exploitation des logiciels a été effectuée à partir d'un fichier constituant une terminologie anglais-français ${ }^{1}$ du commerce international ${ }^{2}$ incluant 324 fiches comportant une moyenne de 110 mots - avec un minimum de 8 mots et un maximum de 155 mots.

Le modèle de fiche (cadre) retenu était le suivant :

a) vedette

b) synonymes (et abréviations)

c) équivalent(s)

d) définition(s)/contexte(s)/explication(s)

- anglais

- français

e) descripteurs de vedette-fiche.

Ce modèle correspond à une structure minimale de fiche satisfaisant aux exigences pratiques des traducteurs et rédacteurs. Rappelons que chacun peut modifier à loisir les nombre et nature des fiches.

Un double impératif avait été fixé en réponse au souci de tester les possibilités de gestion du fichier :

présence simultanée du français et de l'anglais dans le champ des définitions,

- présence obligatoire de descripteurs indiquant : limites de champs d'application ; corrélations interconceptuelles; articulations thématiques. (Descripteurs retenus : $A I R$, MER, RAIL, ROUTE, IMPORTATION, EXPORTATION, ACHETEUR, VENDEUR, RÉCEPTION, EXPÉDITION, CHARGEMENT, DÉCHARGEMENT,

1. Le fichier terminologique prévoyait uniquement deux langues. Il eût été possible, compte tenu de la souplesse de création de cadres de fiches, d'envisager une terminologique multilingue.

2. Le fichier terminologique utilisé avait été réalisé par Évelyne Le GREVELLEC à l'occasion d'un stage dans une entreprise d'import-export. Ce fichier correspondait à ce que l'on pourrait appeler un fichier "fonctionnel" en ce sens que l'auteur y avait seulement consigné, pour chaque vedette, les données qu'elle considérait, subjectivement, comme importantes. Ce fichier n'a pas été modifié et nous l'avons utilisé tel quel afin d'éviter qu'une préconception du fichier ne vienne fausser les objectifs de mise au point des modalités d'exploitation et, en amont, les modalités de sélection des données. Ainsi, certaines fiches sont exhaustive, d'autres fortement lacunaires - toutes les variantes étant possibles entre ces deux extrêmes. 
TRANSPORT, FRAIS, ASSURANCE, DOUANE, BANQUE, LITIGE, DOCUMENT, AGENT, STOCKAGE3.

Chacune des fiches porte ainsi une indexation par séries de descripteurs - ces derniers pouvant être ou non organisés et gérés en thésaurus.

\section{EXPLOITATION/CONSULTATION}

La consultation du fichier par exploitation du logiciel LISCA permet exclusivement la consultation des fiches à partir de la vedette terminologique ou la consultation de la totalité des fiches contenues dans le fichier dans l'ordre alphabétique (le classement s'effectue alors selon la nature de la première lettre du premier composant de l'unité terminologique). La troncature trouve ici une application intéressante puisqu'elle permet d'obtenir toutes les fiches comportant un composant donné dans la vedette terminologique. Ainsi, pour obtenir toutes les fiches comportant LADING, on tapera, à l'interrogation, LADING précédé d'un astérisque. On peut ainsi, pour l'anglais, obtenir toutes les unités terminologiques ayant un même "mot » pour base. Dans la pratique de la troncature, le « mot " peut être tout ensemble de lettres et même un mot orthographique tronqué. Ceci permet d'éviter de prendre en compte, au stade de la constitution du fichier, les corrélations terminologiques : ces dernières sont prises en charge automatiquement par le programme et l'utilisateur remplit ses fiches sans se préoccuper des ' empilages' et de leurs décompositions.

La troncature peut être, dans l'exploitation du fichier par le logiciel BASE, antérieure ou postérieure. Ainsi, pour reprendre un exemple, quiconque désire obtenir toutes les fiches comportant $B I L L$ en composant de vedettes ou unités terminologiques, tapera BILL précédé ET suivi d'un astérisque. Il obtiendra alors les fiches de type $A I R W A Y$ bill en même temps que les fiches de type bill OF LADING. L'exploitation du fichier par le logiciel BASE est, déjà à cet égard, une exploitation plus souple et de plus haut rendement.

On peut, dans l'exploitation du fichier par le logiciel BASE, distinguer trois modalités de consultation qui, dans la pratique, sont concomitantes :

a) Interrogation par " vedettes ": Cette forme d'interrogation s'effectue selon des modalités comparables à celles décrites dans le cas de LISCA. Elle intervient soit par écriture complète de la vedette, soit par troncature. La troncature est utile lorsque l'interrogateur ne peut déterminer avec précision l'extension de l'unité terminologique. Elle permet donc une interrogation de type "aveugle".

Cette forme d'interrogation standard revêt, dans le type de gestion retenu pour la mise au point du programme BASE, une variante qui nous semble présenter de nombreux avantages. En effet, les "vedettes" peuvent être situées dans des champs différents. En clair, la " vedette " réelle — au sens de l'interrogation - peut être l'unité terminologique anglaise ou l'unité terminologique française. Par conséquent, la constitution du fichier n'impose nullement de respecter une suite systématique de type " anglais-français " puisque chaque fiche devient, dans le type de gestion adopté, réversible. Le statut de vedette peut également être dévolu aux synonymes, abréviations, sigles, etc. Il suffit que les champs correspondants soient indexés comme vedettes pour interrogation et la manipulation est globale et automatique.

b) Interrogation par "descripteurs" : BASE accepte, comme toute base de données, une interrogation par descripteurs. Ces descripteurs peuvent être organisés en thé-

3. Dans le fichier construit, les descripteurs n'ont pas été organisés en thésaurus. L'organisation d'un thésaurus est prévue dans le système : dans cette hypothèse, le programme $"$ corrige $»$ tout descripteur fautif aussi bien au moment de l'indexation qu'au moment de l'interrogation. 
saurus mais ceci ne constitue pas une obligation et, par conséquent, le choix des descripteurs peut demeurer partiellement subjectif.

Les interrogations par descripteurs acceptent les combinaisons standard ET/OU/ SAUF. On peut ainsi, par exemple :

- demander toutes les fiches ayant pour descripteur DOUANE

- demander toutes les fiches ayant pour descripteurs DOUANE ET AGENT

- demander toutes les fiches ayant pour descripteur DOUANE OU AGENT

- demander toutes les fiches ayant pour descripteur DOUANE mais pas DOCUMENT (DOUANE SAUF DOCUMENT)

et toutes les combinaisons envisageables compte tenu de la nature des descripteurs d'un fichier donné.

L'interrogation par les descripteurs est de nature " contextuelle":

— interrogation possible lorsque joue uniquement une intuition relative à la nature du contexte ;

- interrogation possible pour balayage d'un secteur ou thème donné.

L'interrogation par descripteurs (interrogation thématique) fait de BASE et des fichiers gérés une source de documentation terminologique ou factuelle.

c) Interrogation par-delà vedettes et descripteurs : Cette dernière forme d'interrogation permet d' "accrocher ", dans n'importe quel champ - et donc AUSSI dans le champ de la définition/contexte/explication - un terme qui n'apparaîtrait ni en vedette ni en descripteur ou encore, dans une autre fiche que la fiche dont elle n'est ni vedette ni descripteur, une unité terminologique donnée. Le rendement de l'interrogation se trouve ainsi maximisé pour deux raisons : toute fiche faisant référence à l'objet de l'interrogation peut être obtenue la mobilisation des fiches permet d'établir les extensions phraséologiques d'une unité terminologique donnée sans que celles-ci soient à prévoir au stade de la constitution des fiches. La seule condition à respecter pour obtenir toutes les fiches pertinentes par exploration de la totalité des champs de fiches ou rubriques est l'encadrement de l'objet de l'interrogation par un caractère @antéposé et un caractère @ postposé.

Si nous avons dissocié les trois types d'interrogations, la dissociation n'a d'autre justification que le souci d'explication : dans la pratique, les trois types d'interrogations sont concomitants sans nécessiter de manipulation spécifique. Ils peuvent par conséquent intervenir selon des séquences déterminées, non pas par l'organisation de la base, mais par les circonstances et objectifs de la consultation. La procédure d'interrogation de BASE est la suivante :

- lancement du programme de consultation (lancement immédiat)

- affichage de "question 1 " (immédiat)

- formulation de question 1 par utilisateur : au choix

- taper l'objet de l'interrogation en "vedette" (recherche ponctuelle)

- taper descripteurs en combinaisons ET/OU/SAUF (recherche thématique)

- taper objet d'interrogation avec troncature (recherche extensive)

- taper MOT entre à(recherche exhaustive)

- affichage "syntaxe correcte" ou "le descripteur X n'existe pas" si question 'mal posée';

affichage de recherche (possibilité de refus ou acceptation des titres de fiches recherchées ou trouvées par programme)

affichage du nombre de références.

- utilisateur peut, (en question 2), affiner question ou poser une seconde question.

Si cheminement satisfaisant ; l'utilisateur demande affichage ou listage des fiches.

L'utilisateur peut conserver ou détruire l'historique des interrogations. 


\section{EFFICACITÉ}

Il va de soi que le rendement d'une terminologie automatisée dépend étroitement des contenus du fichier et de leur organisation. Au-delà de ce rendement absolu, on peut juger de l'efficacité des micro-banques de terminologie en termes de possibilités d'exploitation ou 'souplesse' du système, tant au stade de la création des fichiers qu'au stade des consultations.

Il faut, avant d'aborder les points positifs, accepter de reconnaître le " point faible" des micro-banques de terminologie. Comme leur nom l'indique, ces dernières sont miniaturisées et, par conséquent, leurs capacités de stockage de données sont réduites. On se trouve ainsi contraint, sauf à exploiter un disque dur, à une multiplication des disquettes/fichiers parallèle à la multiplication, en d'autres lieux, des lexiques-papier.

L'inconvénient est aisément surmontable lorsque les domaines traités sont relativement homogènes mais aussi lorsque chaque fichier concerne un secteur donné. Ce même inconvénient serait certes surmonté si l'on utilisait les micro-banques de terminologie en complément des banques de terminologie : le micro-ordinateur servirait ainsi de terminal d'interrogation des banques de données en vraie grandeur, terminal retrouvant sa complète autonomie dans la gestion des fichiers personnels du traducteur/rédacteur/ terminologue/terminographe ${ }^{4}$.

Les avantages offerts par les micro-banques de terminologie sont les suivants :

a) souplesse d'utilisation : les matériels sont transportables et non assujettis à des connexions. Ils sont en outre peu encombrants. Les disquettes sont de rangement facile, aisément échangeables et toujours disponibles.

b) souplesse de définition : chacun peut organiser son fichier comme il l'entend et modifier à volonté les modèles de fiches et les modalités de gestion ou d'exploitation.

c) possibilité de remise à jour constante.

d) possibilité de création de fichiers parallèles. Nous avons, à titre expérimental, adjoint au fichier terminologique proprement dit un fichier élémentaire dans lequel chaque vedette terminologique n'est accompagnée que de ses synonymes et équivalents.

e) modalités de consultation équivalentes à celles des banques de terminologie : si le temps de réponse est supérieur à ce qu'il est pour les "vraies » banques, il importe de souligner, dans le cas présent, la diversité des modalités d'accès aux données pertinentes. BASE permet la recherche terminologique ponctuelle et thématique, mais aussi la recherche phraséologique (par balayage des champs de fiches) ou la recherche documentaire (dans l'organisation thématique des interrogations).

La souplesse de définition des cadres des micro-banques de terminologie ne doit pas faire craindre des effets négatifs : elle ne menace pas les efforts de standardisation et de normalisation. Elle permet au contraire le respect de toute norme puisque le cadre n'est pas préformé. Elle prend néanmoins en compte une donnée évidente : chaque utilisateur a des besoins, objectifs ou finalités spécifiques qui risquent de le conduire, par nécessité et pour lui seul, à adopter des modalités allégées de traitement de terminologies.

Nous pensons que les logiciels utilisés permettent de construire des microbanques de terminologie propres à rendre pleinement justice à la multiplicité des fonc-

4. Les obstacles ne sont pas, en la matière, des obstacles techniques. Ils tiendraient sans doute davantage à des craintes nées des possibilités de copie et peut-être aussi à des tendances (explicables) à la rétention de fiches. 
tions et usages de la terminologie et permettant une exploitation maximale des fichiers terminologiques.

\section{EXPLOITATION PROFESSIONNELLE/EXPLOITATION PÉDAGOGIQUE}

L'exploitation professionnelle des micro-banques de données s'apparente, en modèle réduit, à l'exploitation des banques de terminologie. Il n'est donc pas nécessaire de reprendre la totalité des formes d'utilisation envisageables. Nous nous contenterons de préciser les différences essentielles entre les deux types de «banques » en insistant, d'une part, sur le fait que la gestion est, dans le cas des micro-banques, gestion individuelle et, d'autre part, sur le fait que le modèle de micro-banque proposé se prête, par excellence, à la documentation de type phraséologique et à la documentation thématique.

La documentation phraséologique est possible par modification des modèles (cadres) de fiches. Ce type de documentation est utile au traducteur et, plus encore, au rédacteur, et l'on peut aisément concevoir un fichier dont les vedettes soient des unités phraséologiques et les descripteurs des modalités d'indexation par registre, finalité, performatifs, niveau de langue, aire géographique, et ainsi de suite au gré de l'utilisateur ${ }^{5}$.

La documentation thématique relève directement de l'exploitation des fichiers par BASE avec interrogation par descripteurs « thématiques » ou, plus encore, par combinaisons de descripteurs selon les voies du thésaurus.

Les micro-banques de terminologie ouvrent, parallèlement, des voies nouvelles dans la définition des programmes de formation : elles peuvent rapidement - toujours en raison de leur grande souplesse de définition et d'utilisation - devenir l'instrument d'apprentissages divers.

Le premier apprentissage est celui qui porte sur l'initiation à la manipulation de l'outil informatique à des fins spécifiques. L'initiation interviendrait alors en parfaite autonomie : pas de connexion ; coût de fonctionnement pratiquement nul ; disponibilité constante des programmes ; possibilité de création individuelle ou collective de modèles ou cadres de fiches; acquisition des modalités et procédures de gestion de fichiers.

Au-delà de cette initiation, il faut retenir les éléments d'apprentissage terminographique/terminologique essentiels à toute formation de traducteurs ou de traducteursrédacteurs.

On pourrait faire valoir, sans nul doute, que la fiche-papier a du bon et qu'elle ne sera pas détrônée de si tôt. Cependant, rien n'interdit d'utiliser les possibilités offertes par le modèle de micro-banque de terminologie proposé pour :

- éclairer la nature des contraintes imposées au terminographe, tant en vertu des ' normes' régissant ses activités qu'en vertu des contraintes nées du fait qu'une terminologie est terminologie morte si elle n'est pas accessible ;

- mettre en évidence les incidences d'un choix déterminé de modèle de cadre sur la sélection des données pertinentes;

- mettre en évidence les variations de pertinence de données terminologiques en fonction de modes divers d'interrogation conditionnés ou déterminés par des objectifs spécifiques de documentation terminologique. En clair, montrer que

5. Le CRAIE - Centre de recherche sur les applications de l'informatique à l'enseignement - à l'Université de Haute-Bretagne à Rennes (France) se concentre actuellement sur un projet de constitution et exploitation de fichiers documentaires pour rédacteurs constitués et gérés par les mêmes logiciels que ceux décrits ici. Ces fichiers sont un fichier phraséologique et un fichier documentaire. Les indexations des unités phraséologiques s'effectuent par les descripteurs suivants : aire géographique, registre, niveau de langue, système de valeur, domaine de stéréotypie, implications, finalités, intentions, performatif, cadres. 
les terminologies se prêtent à des utilisations différenciées selon la nature des tâches à la réalisation desquelles elles contribuent ;

- mettre en évidence les statuts et fonctions respectifs des différents éléments présents dans une fiche de terminologie ou dans la totalité du fichier;

- apprendre la notion de descripteur et, surtout, prendre conscience de la fonction spécifique des descripteurs. Les micro-banques de terminologie permettent une évaluation immédiate de la pertinence, de la fiabilité, de l'exhaustivité et de la non-redondance des descripteurs puisque l'interrogation dénoncera immédiatement toute carence - toute erreur de description conduira à des "accidents de consultation".

Naturellement, les terminologues n'ont pas attendu la mise au point des banques de terminologie pour s'interroger sur leur pratique. Cependant, l'utilisation de microbanques de terminologies - chacune étant construite par un étudiant - permet de poussr cette réflexion et de rendre TANGIBLES les contraintes, impératifs ou restrictions auxquels ne peut échapper la terminographie. Les micro-banques de terminologie doivent permettre une démonstration immédiate des intercorrélations entre la nature des données intégrées au fichier et les niveaux de rendement du fichier selon les objectifs de la consultation. Elles sont à la fois un moyen de produire des fichiers efficaces puisqu'elles peuvent s'accommoder de toutes variations de modèles ou cadres - et un moyen de tester l'efficacité des fichiers - puisqu'elles autorisent des interrogations ou consultations adaptées à chaque finalité d'exploitation que l'on peut imaginer en matière de terminologie.

\section{CONCLUSION}

Les logiciels permettant création et gestion des micro-banques de terminologie ne doivent pas fatalement conduire à une réduction des contenus de fiches ou à une réduction des possibilités d'exploitation de fichiers. Toutes choses étant égales par ailleurs nombre de rubriques et nature des données - et après levée de l'hypothèque due à la taille réduite des fichiers, les micro-banques de terminologie peuvent rendre les plus grands services, individuellement (dans l'hypothèse d'un micro-ordinateur autonome) ou collectivement (dans l'hypothèse d'un micro-ordinateur en fonction de terminal ou d'échanges de fichiers) aux traducteurs, rédacteurs ou terminographes. Elles peuvent sans doute devenir un outil privilégié des apprentissages dans le cadre de programmes de formation. Elles doivent, à brève échéance, devenir aussi l'instrument privilégié d'une recherche s'interrogeant sur les corrélations entre la nature de la terminologie, la nature de ses déterminants et la nature de ses fonctions.

\section{QUELQUES CARACTÉRISTIQUES DE LA BASE GÉRANT LA MICRO-BANQUE}

- fonctionne sur micro-ordinateurs 8 bits.

- le nombre de fiches est libre dans les limites suivantes:

- programmes occupent $20 \%$ de la disquette double face

- volume d'information résiduelle en mémoire inférieur à $5 \%$

- le temps de recherche n'est pas lié au nombre de fiches

- la base comporte, sur micro 8 bits, un THESAURUS complet qui gère :

- l'appartenance au domaine (ou appartenance aux secteurs)

- les termes associés à un descripteur

- les quasi-synonymes

- la signalisation des non-descripteurs

- les relations de termes à termes génériques et à termes spécifiques SANS LIMITA-

TION DE NOMBRE ET DE TAILLE DES DESCRIPTEURS. 